\title{
Análisis técnico y económico para la incorporación de facts en sistemas de potencia*
}

\author{
José D. Gómez J.** \\ Guillermo E. Vinasco M.** \\ Jesús M. López-Lezama****
}

Recibido: 10/03/2016 - Aceptado: 13/12/2016

DOI: $10.22395 /$ rium.v16n31a12

\begin{abstract}
Resumen
En este artículo se presenta una metodología para el análisis técnico y económico de la incorporación de dispositivos Facts en sistemas eléctricos de potencia. La metodología propuesta consta de una fase determinista y una estocástica que consideran aspectos de factibilidad técnica y económica, respectivamente. La primera fase se basa en un modelo de programación no lineal binario mixto desarrollado en AMPL que pretende minimizar la generación de seguridad dada la ubicación de un dispositivo Facts. La segunda fase utiliza un modelo energético basado en programación dual dinámica estocástica (SDDP) que determina las relaciones beneficio/costo de la incorporación de Facts para diferentes series hidrotérmicas. Para ilustrar la aplicabilidad de la metodología propuesta se presenta un estudio de caso en el sistema eléctrico colombiano considerando la incorporación de un Statcom en la costa atlántica. Adicionalmente, se muestra que la solución propuesta resuelve problemas de inestabilidad oscilatoria de tensión identificados en escenarios mínimos de generación ante contingencias críticas.
\end{abstract}

Palabras clave: dispositivos Facts; optimización; sistemas de potencia.

Artículo no derivado de investigación.

** Estudiante de Ingeniería Eléctrica, Departamento de Ingeniería Eléctrica, Universidad de Antioquia, Calle 67 No53-108, Medellín, Colombia. josegomezj280@gmail.com

*** Ph.D en Ingeniería, Interconexión Eléctrica S.A E.S.P. Calle 12 Sur N 18-168, Medellín, Antioquia. gevinasco@ intercolombia.com

**** Ph.D en Ingeniería, Profesor del Departamento de Ingeniería Eléctrica, Universidad de Antioquia, Calle 67 №53-108, Medellín, Colombia. jesusmarialopezl@gmail.com 


\title{
Technical and economic analysis for the incorporation of Facts in power systems
}

\begin{abstract}
This article shows a methodology for the technical and economic analysis of the incorporation of Facts devices in electrical power systems. The methodology proposed consists of a deterministic stage and a stochastic stage which involve aspects of technical and economic feasibility, respectively. The first stage is based on a non-linear binary mixed programming model developed at AMPL in order to minimize generation of safety given the location of a Facts device. The second stage employs an energetic model based on a stochastic dynamic dual programming (SDDP) which determines the cost-benefit ratios of the incorporation of Facts for different hydrothermal series. In order to illustrate the applicability of the methodology proposed, a case study is shown in the Colombian electrical system, taking into account the incorporation of a Statcom in the Atlantic Coast. Additionally, it is shown that the solution proposed resolves oscillatory instability problems of power, identified in minimum generation scenarios before critical contingencies.
\end{abstract}

Keywords: facts devices; optimization; power systems.

\section{Análise técnica e econômica para a incorporação de Facts em sistemas de potência}

\begin{abstract}
Resumo
Neste artigo, apresenta-se uma metodologia para a análise técnica e econômica da incorporação de dispositivos Facts em sistemas elétricos de potência. A metodologia proposta consta de uma fase determinista e uma estocástica que consideram aspectos de factibilidade técnica e econômica, respectivamente. A primeira fase baseia-se num modelo de programação não linear binário misto, desenvolvido em AMPL que pretende minimizar a geração de segurança dada a localização de um dispositivo Facts. A segunda fase utiliza um modelo energético baseado em programação dual dinâmica estocástica (SDDP) que determina as relações custobenefício da incorporação de Facts para diferentes séries hidrotérmicas. Para ilustrar a aplicabilidade da metodologia proposta, apresenta-se um estudo de caso no sistema elétrico colombiano considerando a incorporação de um Statcom no litoral atlântico colombiano. Além disso, mostra-se que a solução proposta resolve problemas de instabilidade oscilatória de tensão identificados em cenários mínimos de geração ante contingências críticas.
\end{abstract}

Palavras-chave: dispositivos Facts; optimização; sistemas de potência. 


\section{INTRODUCCIÓN}

En el planeamiento de los sistemas eléctricos de potencia (SEP) se busca predecir requerimientos futuros de la red (ubicación y tipo de demandas futuras, requerimientos de generación, etc.) para mantener un servicio económico (con menores costos para los usuarios finales) y seguro. Esto implica limitar transferencias de potencia y uso de generación de seguridad. Esta última se define como la generación requerida en un área, para evitar problemas de sobrecargas y de tensiones tanto en caso base como ante contingencias $\mathrm{N}-1$. Las restricciones de transporte de los SEP se pueden aliviar con nuevos proyectos de transmisión, pero las exigencias ambientales, sociales y económicas para su construcción son cada vez más severas [1,2]. Algunos problemas como estabilidad y límites de generación de seguridad tienen alternativas de solución vía instalación de dispositivos de compensación controlados por electrónica de potencia, llamados dispositivos Facts [3]. El reto para el planeador del sistema consiste en justificar técnica y económicamente los beneficios de instalar Facts, mostrando que estos son mayores que la inversión requerida.

Con el crecimiento y desregulación de los sistemas eléctricos de potencia, surgieron problemáticas de operación y nuevos requerimientos para el control del flujo de potencia y el cumplimiento de criterios de confiabilidad [3]. Los Facts permiten redistribuir el flujo de potencia y aprovechar la capacidad de transmisión no utilizable en actuales instalaciones por problemas de estabilidad; así se logran beneficios al aplazar inversiones para la construcción de nuevos corredores de transmisión, o reducir plantas de generación destinadas como seguridad para un área débil [4].

Dentro de los dispositivos Facts se encuentran los SVC (Static Var Compensators), que se instalan en paralelo para generar o absorber potencia reactiva. Estos dispositivos intercambian corriente reactiva capacitiva o inductiva [5]. Por otro lado, los Statcom (Static Synchronous Compensator) son un tipo de Facts más avanzado, cuya corriente reactiva de intercambio no depende de la tensión a la que está conectado [6]. El compensador serie controlado por tiristores (sigla-TCSC) es un Facts que permite un ajuste rápido y continuo de la impedancia en una línea de transmisión, y su estructura básica consta de un banco de condensadores en paralelo con un reactor TCR (Thyristor controlled reactor) [7]. Otro tipo de Facts son las baterías BESS (Battery Energy Storage System) las cuales tienen la capacidad de acumular energía y entregarla en forma rápida al sistema permitiendo que las unidades de generación permanezcan en rangos nominales [8]. Los costos de estos dispositivos dependen de factores como su potencia nominal y tecnología, siendo más onerosos aquellos dispositivos dinámicos que controlan potencia reactiva inductiva y capacitiva como los Statcom. 
En la literatura técnica se encuentran numerosos estudios para la ubicación óptima de dispositivos Facts. Se destaca la aplicación de técnicas metaheurísticas como el enjambre de partículas [9], algoritmos de búsqueda gravitacional [10], y algoritmos genéticos [11], entre otros. Una revisión sobre metodologías aplicadas para la ubicación óptima de dispositivos Facts puede ser consultada en [12]. La mayoría de los métodos mencionados anteriormente buscan encontrar valores de muchas variables de optimización de manera simultánea, en un único problema de optimización que es de naturaleza no lineal, no convexa y multimodal. Lo anterior lleva a modelos altamente complejos que pueden requerir tiempos de cálculo prohibitivos cuando se aplican a sistemas de potencia reales. La contribución principal de este trabajo radica en la aplicabilidad de la metodología para casos reales y no en la ubicación óptima de dispositivos desde el punto de vista de programación matemática. Esto se fundamenta en el hecho de que en muchas ocasiones el operador o planeador de red conoce las posibles ubicaciones de los dispositivos Facts, pero debe utilizar herramientas técnicas y económicas para tomar una decisión definitiva sobre su ubicación. Este último aspecto es el abordado en el artículo. Adicionalmente, vale la pena mencionar que en Colombia hay pocos dispositivos de este tipo y no existe una metodología estándar para su ubicación (básicamente se depende de la experticia del operador de red), por lo que la metodología propuesta puede ser implementada para evaluar la factibilidad de inversiones posteriores.

La metodología propuesta consta de una fase determinista y una estocástica para considerar aspectos de factibilidad técnica y económica, respectivamente. En la fase 1 se utiliza un modelo de programación no lineal binario mixto desarrollado en AMPL ( $A$ Mathematical Programming language) [13]. La función objetivo consiste en minimizar la generación de seguridad considerando un modelo $\mathrm{AC}$ de la red de transmisión y contingencias N-1 [14]. En el modelo no se tiene en cuenta la ubicación de los dispositivos Facts como variable de decisión. En lugar de esto se supone que el operador de red ha identificado previamente las tecnologías de Facts y barras candidatas, por lo que se hace una evaluación exhaustiva de estas posibles soluciones.

En la fase 2 se aplica un modelo energético de programación dual dinámica estocástica (SDDP) [15], para calcular el impacto en costos operativos debido a la instalación de Facts. El modelo se ejecuta para cien series hidrotérmicas obteniendo las relaciones beneficio / costo para cada una de estas, lo que da un indicio de la viabilidad económica del proyecto.

Se realizaron varias pruebas para la ubicación de Facts en el área Caribe del sistema eléctrico colombiano. La validez de los resultados del modelo AMPL se corroboró mediante simulaciones en DigSilent Power Factory [16], incluyendo el hecho de que la instalación de un dispositivo Facts identificado por el modelo, evita una inestabilidad oscilatoria de tensión en la Costa Atlántica para 2018. 


\section{METODOLOGÍA}

En la figura 1 se ilustra la metodología propuesta. La primera fase (determinista) corresponde a la optimización no lineal realizada en AMPL, y la fase 2 corresponde a la optimización estocástica realizada en SDDP.

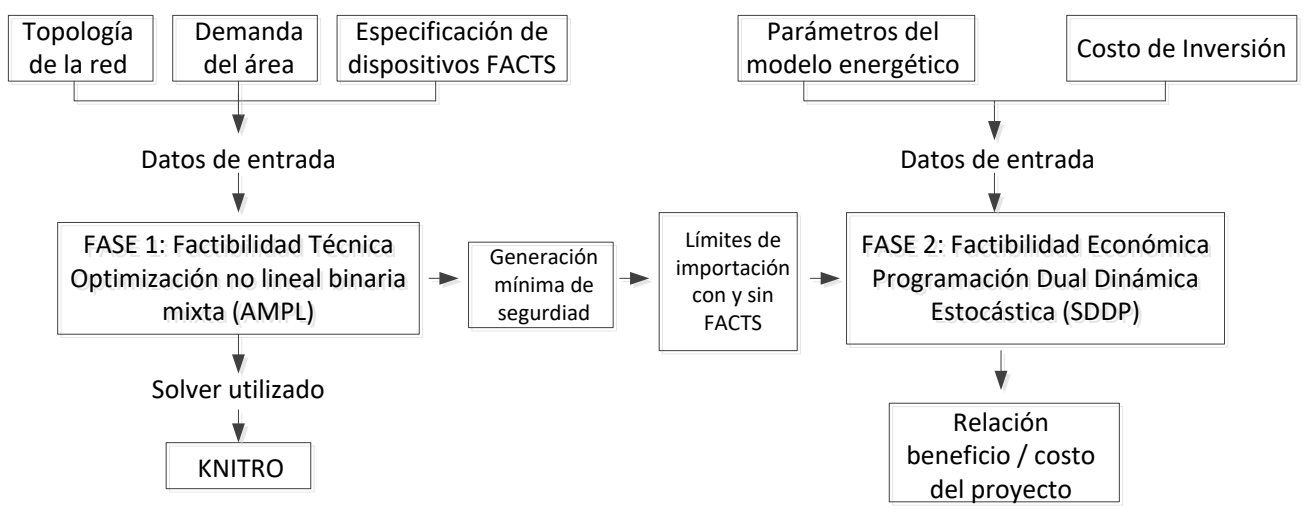

Figura 1: metodología propuesta.

Fuente: elaboración propia

\subsection{Fase 1. Minimización de generación de seguridad}

En esta fase la función objetivo consiste en minimizar la generación de seguridad. Para ello se utiliza un flujo de potencia óptimo AC, combinado con un unit commitment. Las ubicaciones para Facts son limitadas. En esta fase se realizan varias corridas del modelo para barrer las ubicaciones factibles; las variables binarias deciden qué máquinas utilizar como generación de seguridad; las variables continuas indican: cantidades de generación requeridas (MW, MVAr), taps en los transformadores y punto de operación del dispositivo Facts.

En el modelo se definen los conjuntos de ramas, nodos y contingencias como R, $\mathrm{Ny} C \mathrm{~T}$, respectivamente. En este caso el índice indica el caso base (sin contingencias) mientras indica las contingencias definidas para la red. La función objetivo se ilustra en la ecuación (1), donde son las variables binarias que determinan la operación de la generación, tanto para el caso base, como para contingencias. Por otro lado, es la potencia generada en el caso base.

$$
\min G e n=\sum_{\forall n \in N} u_{n} * p g_{n, c t=0}
$$

La función objetivo está sujeta a las restricciones de balance de potencia activa, dadas por las ecuaciones (2) y (3), respectivamente. 


$$
\begin{gathered}
p g_{i, c t}-P d_{i}-v_{i, c t} \sum_{j \in b} v_{j, c t}\left[g_{i j} \cos \theta_{i j}+b_{i j} \sin \theta_{i j}\right]+\text { pfacts }_{i, c t}=0 \\
\forall c t \in C T, \forall i, j \in N \\
q g_{i, c t}-q d_{i}-v_{i, c t} \sum_{j \in b} v_{j, c t}\left[g_{i j} \sin \theta_{i j}-b_{i j} \cos \theta_{i j}\right]+q f a c t s_{i, c t}=0 \\
\forall c t \in C T, \forall i, j \in N
\end{gathered}
$$

Donde

Ij: índices que pertenecen al conjunto de nodos $N$.

$p g_{i, c t} q g^{i, c t}$ : generación en la barra $i$ ante la contingencia $c t$.

$p d_{i}, q d_{i}$ : potencias activa y reactiva demandadas en el nodo $i$, respectivamente

$v_{i, c t}:$ tensión asociada al nodo $i$ en la condición $c t$.

$\theta_{i j}$ : diferencia angular entre los nodos $i$ y $j$.

pfacts $_{i, c}$ qfacts $s_{i, c t}$ : potencias activa (en caso de baterías) y reactiva inyectada por un Facts en paralelo conectado al nodo $i$

$g_{i j}, b_{i j}$ : parte real e imaginaria de la posición $i, j$ de la matriz de admitancia nodal.

Para el cálculo de la matriz de admitancia nodal se utilizan las ecuaciones (4) y (5).

$$
\begin{gathered}
g=\left\{g_{i j}=-G_{i j} *\left(N_{r}-N_{c t}\right)\right. \\
b=\left\{\begin{array}{l}
g_{i i}=\sum_{j \in n} G_{i j} *\left(N_{r}-N_{c t}\right) \\
b_{i i}=B_{i j}^{s h}+b_{i, c t}^{s v c}+B_{i}^{s h}+\sum_{j \in n} B_{i j} *\left(N_{r}-N_{c t}\right)
\end{array}\right.
\end{gathered}
$$

Donde:

$G_{i j}, B_{i j}, B_{i j}^{s h}:$ conductancia, susceptancia y susceptancia en derivación de las diferentes ramas entre los nodos $i$ y $j$.

$b_{i, c t}^{s v c}$ : susceptancia variable de un SVC conectado al nodo $i$ en contingencia $c t$.

$B_{i}^{s h}$ : susceptancia fija de compensación conectada al nodo $i$.

$N_{r}:$ número de ramas con iguales características en paralelo.

$N_{c l}:$ número de ramas que fallan en contingencia. 
El modelo considera límites en la generación de potencia activa y reactiva de las máquinas del sistema; para indicar si están o no en servicio se utiliza una variable binaria, como se indica en las ecuaciones (6) y (7).

$$
\begin{gathered}
\operatorname{PMIN}_{n} * u_{n} \leq p g_{n, c t} \leq \operatorname{PMAX}_{n} * u_{n}, \forall c t \in C T, \forall n \in N \\
\operatorname{QMIN}_{n} * u n_{n} \leq q g_{n, c t} \leq Q M A X_{n} * u_{n}, \forall c t \in C T, \forall n \in N
\end{gathered}
$$

Donde:

$P M I N_{n}, P M A X_{n}$ : límites inferior y superior de generación de potencia activa, para un generador conectado en el nodo $n$.

$Q M I N_{n}, Q M A X_{n}$ : límites inferior y superior de generación de potencia reactiva, para un generador conectado en el nodo $n$.

Las tensiones de los nodos se deben mantener en límites mínimos y máximos. En Colombia estos límites son diferentes para barras de diferentes tensiones y si la red está en estado normal o bajo contingencia. Esta restricción está dada por la ecuación (8).

$$
V M I N_{n} \leq v_{n, c t} \leq V M A X_{n}, \forall c t \in C T, \forall n \in N
$$

Donde:

$V M I N_{n}, V M A X_{n}$ : límites mínimos y máximos de tensión para los nodos $n$ del sistema. Estos cambian entre estado normal $(c t=0)$ y bajo contingencias $(c t>0)$.

Se consideran límites en la potencia total (MVA) de elementos a: $110 \mathrm{kV}, 220 \mathrm{kV}$ y $500 \mathrm{kV}$, con una capacidad para el caso base $(c t=0)$ y la sobrecarga permitida ante contingencias de la red $(c t>0)$ como indicado en las ecuaciones (9) y (10).

$$
\begin{gathered}
s_{r, c t} \leq S M A X_{r}, \forall r \in R, c t=0 \\
s_{r, c t} \leq S=A X C_{r}, \forall r \in R, c t=0
\end{gathered}
$$

Donde:

$S_{r, c}:$ flujo de potencia aparente por las ramas $r$.

$S M A X_{r}, S M A X C_{r}$ : potencia máxima permitida para el caso base y bajo contingencias, respectivamente.

Para los taps de los transformadores, tanto en caso base como en contingencias, se definen límites máximos y mínimos de operación. Bajo contingencia los taps deben mantener los valores del caso base.

$$
\text { TAPMIN }_{r} \leq \text { tap }_{r} \leq \text { TAPMAX }_{r}, \forall r \in R
$$


Donde:

tap $_{r}$ : variable continua que representa las variaciones en los taps de los transformadores.

$\operatorname{TAPMIN}_{r}$, TAPMAX $_{r:}$ límites mínimo y máximo de los taps, respectivamente.

De acuerdo con su tecnología, los Facts permiten inyección de potencia activa o reactiva y poseen límites en susceptancia $\left(b_{n, c t}^{\text {svc }}\right)$, corriente de compensación $\left(i_{n, c t}^{\text {statcom }}\right)$, o potencias reactiva $\left(q f_{a c t s}, c\right)$ y activa $\left(\right.$ pfacts $\left._{i, c}\right)$. Estas restricciones, según el tipo de dispositivo (SVC, Statcom o batería BESS), se indican en las ecuaciones (12) a (16).

$$
\begin{gathered}
B M I N_{n}^{s v c} \leq b_{n, c t}^{\text {svc }} \leq B M A X_{n}^{s v c} \forall n \in N \\
-I M A X_{n} \leq i_{n, c t}^{\text {statcom }} \leq I M A X_{n} \forall n \in N, \forall c t \in C T \\
q_{n, c t}^{\text {statcom }}=i_{n, c t}^{\text {statcom }} * v_{n, c t} \forall n \in N, \forall c t \in C T \\
\operatorname{PMIN}_{n}^{\text {bess }} * u_{n} \leq p_{n, c t}^{\text {bess }} \leq P M A X_{n}^{\text {bess }} * u_{n}, \forall c t \in C T, \forall n \in N \\
\operatorname{QMIN}_{n}^{\text {bess }} * u_{n} \leq Q_{n, c t}^{\text {bess }} \leq Q M A X_{n}^{\text {bess }} * u_{n}, \forall c t \in C T, \forall n \in N
\end{gathered}
$$

Donde:

$B M I N_{n}^{s v c}, B M A X_{n}^{s v c}$ : límites mínimo y máximo de la susceptancia de un SVC conectado al nodo $n$.

$I M A X_{n}$ : límite de inyección en corriente de un Statcom conectado en el nodo $n$.

$P M I N_{n}^{\text {bess }}, P M A X_{n}^{\text {bess }}$ : límites en potencia activa de batería BESS.

$Q_{M I N}^{\text {bess }}, Q M A X_{n}^{\text {bess }}$ :límites en potencia reactiva de batería BESS.

Las ecuaciones (12), (13), (14) y (15) se ajustan para limitar los dispositivos Facts en un $20 \%$ de su capacidad para el caso base y queda el resto de capacidad como reserva dinámica ante contingencias.

Las ecuaciones (17) y (18) limitan la potencia total de las compensaciones serie del sistema y la susceptancia de compensación, respectivamente.

$$
\begin{gathered}
\operatorname{scs}_{r, c t} \leq \operatorname{SMAXCS}_{r}, \forall r \in R, c t \in C T \\
B_{n}^{s h} M I N \leq b_{n}^{s h} \leq B_{n}^{s h} M A X, \forall n \in N
\end{gathered}
$$




\section{Donde}

$s c s_{r, c t}:$ potencia que circula por la compensación serie para la rama $r$ en contingencia $c t$.

$S M A X C S_{r}$ : límite máximo de potencia para la rama $r$

$B_{n}^{s h} M I N, B_{n}^{s h} M A X$ : límites inferior y superior para la compensación paralela conectada al nodo $n$.

Para elegir la generación, el modelo AMPL posee restricciones en los ciclos combinados. Mediante las variables binarias nombradas $\left(u_{1}, u_{2}, u_{3}\right.$ etc.), se despachan las unidades de vapor después del despacho de las unidades de gas correspondientes.

El modelo AMPL dado por las ecuaciones (1) a (18) es no lineal binario mixto. En este caso se utilizó el solver KNITRO del NEOS Server, servicio ofrecido por la Universidad de Wisconsin, para dar solución al modelo de esta fase. Dado que el modelo es determinístico (para la misma información de entrada los resultados son idénticos) se realizan varias corridas considerando diferentes disponibilidades para plantas de un área, y así se obtienen diferentes escenarios de generación de seguridad.

\subsection{Fase 2: Cálculo de beneficios económicos con SDDP}

SDDP es un modelo de despacho hidrotérmico con representación de la red de transmisión, utilizado por el operador del sistema colombiano en estudios operativos de corto, mediano y largo plazo. El modelo calcula la política operativa estocástica de mínimo costo de un sistema hidrotérmico teniendo en cuenta detalles operativos, representación de los mercados e incertidumbre hidrológica [17].

En la fase 2 los resultados de la fase 1 alimentan el modelo SDDP (ver figura 1), que calcula los costos de operación con y sin Facts, para un horizonte de 25 años, con tasa descuento del 10\%. La diferencia entre los valores anteriores son los beneficios del Facts. El modelo considera: red de transmisión, detalles operativos de las plantas hidroeléctricas (incertidumbre hidrológica, balance hídrico, límites de turbinado y almacenamiento, volúmenes de seguridad, vertimiento, filtración, etc.), detalles de las plantas térmicas (restricciones de generación debidas a contratos, curvas de eficiencia cóncavas y convexas, restricciones de consumo de gas, térmicas bi-combustible, etc.).

Dado que SDDP utiliza flujo de potencia DC, los límites de estabilidad en tensión le son reflejados como límites de importación los cuales son obtenidos de los resultados del modelo AMPL por medio de la ecuación (19).

$$
\text { Límiteimportación } A M P L \geq \text { Demandatotal-generación del área }
$$


En este caso $n$ es el número del escenario futuro de generación obtenido por SDDP. Cada "Limite Importación AMPL" es un dato de entrada que depende si se instaló el Facts y su localización. Los costos se comparan al cambiar en SDDP "Limite importación AMPL" por la ubicación de Facts; las reducciones son los beneficios. Para cada ubicación de Facts, se calculan cien series con su relación beneficio / costo.

\section{PRUEBAS Y RESULTADOS}

Los resultados que se describen a continuación corresponden a cálculos con datos reales para el sistema colombiano suministrados por el operador de red.

\subsection{Descripción del sistema de prueba}

La red de prueba para la metodología es el sistema eléctrico colombiano, conformado por dos áreas principales: la Costa Atlántica (al norte, con generación térmica) y el interior de Colombia (con generación hidráulica) como se indica en la Figura 2. En 2018 las dos áreas estarán unidas por cuatro circuitos de $500 \mathrm{kV}$, con capacidad térmica de 1300 MW cada uno.

A pesar de que teóricamente la capacidad de transferencia desde el interior de Colombia hacia la Costa Atlántica es de 4x1300 MW, según estudios para 2018, la estabilidad de tensión ante contingencias N-1 en los circuitos de $500 \mathrm{kV}$ no permitirá transferir más de 2060 MW. Por lo tanto, para una demanda máxima esperada en 2018 de 2370 MW en la Costa Atlántica, sería obligatorio generación de seguridad, con plantas térmicas que presentan bandas prohibidas de generación (mínimos técnicos), y ciclos combinados de un orden particular para despacho. Por ello, la cantidad mínima de generación a despachar en la Costa Atlántica, podría superar la diferencia entre la demanda total (2370 MW) y la transferencia máxima a 2018 (2060 MW), produciendo sobrecostos al sistema. Estos sobrecostos se pueden aminorar con un nuevo circuito de conexión a $500 \mathrm{kV}$, o mediante la instalación de dispositivos Facts en la Costa Atlántica. En el presente artículo se explora esta última opción por sus menores costos y menor tiempo de implementación.

\subsection{Fase 1: Minimizar generación de seguridad}

Inicialmente se realiza un diagnóstico del sistema sin Facts mediante simulaciones en el software DigSilent Power Factory considerando contingencias N-1 y restricciones de red. El criterio de seguridad N-1 debe tenerse en cuenta según la regulación colombiana (Resolución CREG 025 de 1995). En esta primera etapa se encontró que la salida de la línea La Loma-Ocaña (de $500 \mathrm{kV}$ ) es la que más generación de seguridad requiere $(763 \mathrm{MW})$. Esto evidencia la necesidad de un Facts que inyecte potencia 


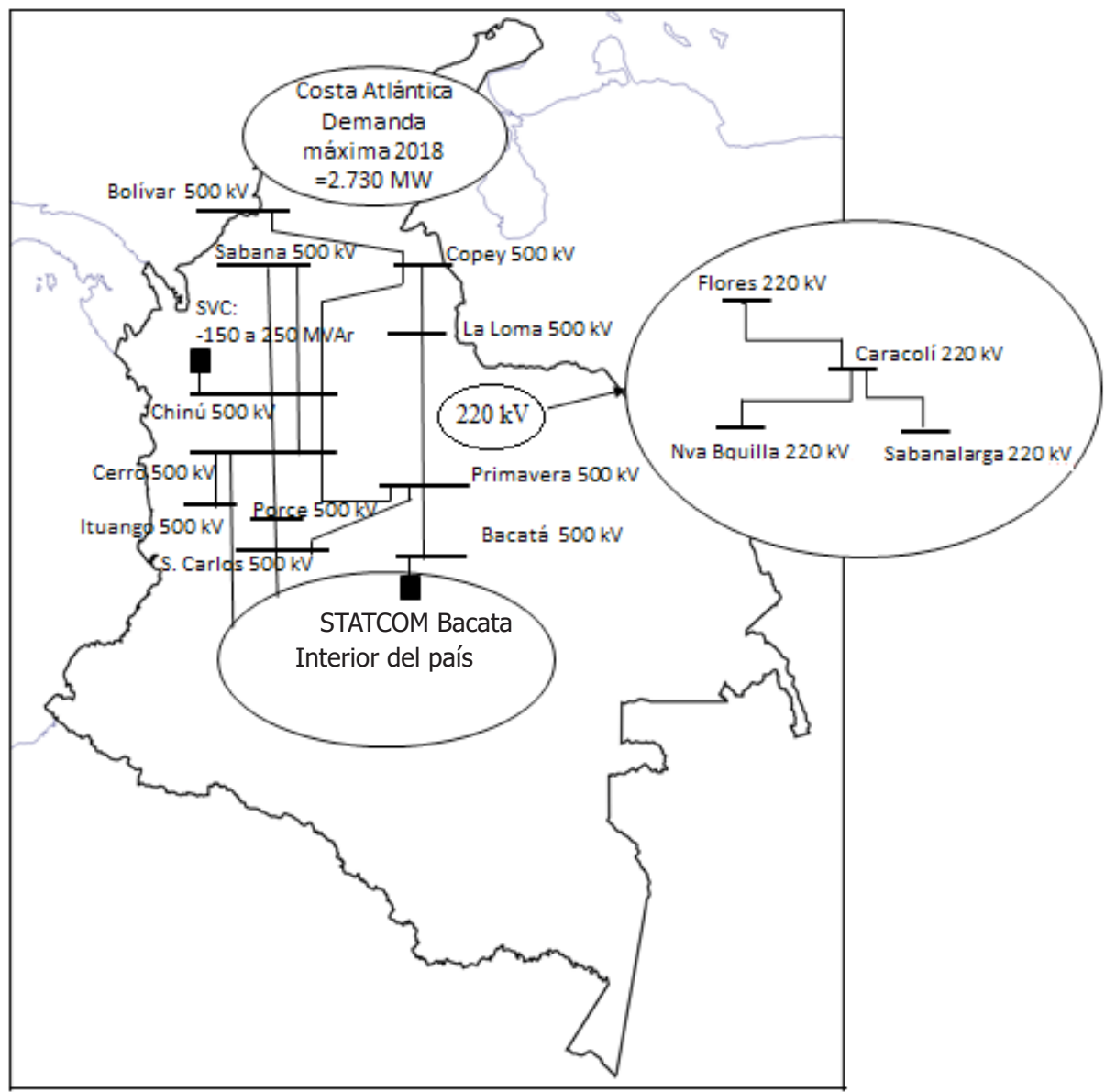

Figura 2: esquema sistema de potencia colombiano a 2018.

Fuente: elaboración propia

reactiva para cumplir con todas las restricciones (especialmente bajo contingencias). Si bien el modelo propuesto permite decidir entre SVC, Statcom o batería BESS, por tecnología superior se supone la inversión de un Statcom, probando ubicaciones factibles en las siguientes barras: Copey 220 kV, Copey 500 kV, Cuestecitas 220 kV y Caracolí $220 \mathrm{kV}$.

En la tabla 1 se muestra la generación de seguridad necesaria ante la contingencia más crítica (La Loma-Ocaña $500 \mathrm{kV}$ ), para las cuatro posibles ubicaciones de compensación dinámica. Se observa que hay menor generación de seguridad (711 MW) con la instalación del Statcom en Caracolí $220 \mathrm{kV}$. En la tabla 2 se presenta la inyección de potencia reactiva del Statcom para cada ubicación. 
Tabla 1: generación de seguridad requerida en contingencia La Loma-Ocaña 500 kV.

\begin{tabular}{|c|c|c|c|}
\hline $\begin{array}{c}\text { Statcom en Copey } \\
220 \mathrm{kV}\end{array}$ & $\begin{array}{c}\text { Statcom en Copey } \\
500 \mathrm{kV}\end{array}$ & $\begin{array}{c}\text { Statcom en Cuestecitas } \\
220 \mathrm{kV}\end{array}$ & $\begin{array}{c}\text { Statcom en Caracoli } \\
220 \mathrm{kV}\end{array}$ \\
\hline $760 \mathrm{MW}$ & $764 \mathrm{MW}$ & $744 \mathrm{MW}$ & $711 \mathrm{MW}$ \\
\hline
\end{tabular}

Fuente: elaboración propia

Tabla 2: inyección del Statcom en caso base y contingencia (MVAr).

\begin{tabular}{|l|c|c|c|c|}
\cline { 2 - 5 } \multicolumn{1}{c|}{} & $\begin{array}{c}\text { Statcom en Copey } \\
220 \mathrm{kV}\end{array}$ & $\begin{array}{c}\text { Statcom en Copey } \\
500 \mathrm{kV}\end{array}$ & $\begin{array}{c}\text { Statcom en Cueste- } \\
\text { citas } 220 \mathrm{kV}\end{array}$ & $\begin{array}{c}\text { Statcom en Cara- } \\
\text { colí } 220 \mathrm{kV} \\
\text { Caso Base }\end{array}$ \\
\hline Contingencia & 60 & 41 & 41 & 43 \\
\hline
\end{tabular}

Fuente: elaboración propia

El modelo en AMPL permite crear varios escenarios operativos en los que participan diferentes generadores. A continuación, se describen tres escenarios considerando el Statcom en la barra Caracolí a $220 \mathrm{kV}$. El escenario 2 corresponde al de mínima generación de seguridad, ya encontrado y los escenarios 1 y 3 se construyen a partir de este. La creación de escenarios permite no ligar la operación del área a un mismo número y tipo de unidades. Es decir, el modelo identifica el escenario con mínima generación de seguridad; sin embargo, es posible recrear otras situaciones operativas al inhabilitar (o habilitar) algunas plantas en este escenario, pues en la operación real, es posible que algunas plantas no siempre estén disponibles.

Los tres escenarios se crearon combinando disponibilidad de las plantas del área Caribe. En la tabla 3 se muestra la generación de seguridad correspondiente para cada escenario. Note que el escenario 2 es el de mínima generación de seguridad y, como era de esperarse, los escenario 1 y 3 representan soluciones de menor calidad.

Tabla 3: generación de seguridad requerida con y sin Statcom.

\begin{tabular}{|c|c|c|c|c|c|}
\hline \multicolumn{3}{|c|}{ Sin Statcom } & \multicolumn{3}{c|}{ Con Statcom } \\
\hline Escenario & MW & MVAr & Escenario & MW & MVAr \\
\hline 1 & 810 & 220 & 1 & 714 & 38 \\
\hline 2 & 712 & 252 & 2 & 565 & 92 \\
\hline 3 & 674 & 204 & 3 & 619 & 17 \\
\hline
\end{tabular}

Fuente: elaboración propia 
- Para el escenario 1 hay disminución en generación de seguridad de 96 MW y 182 MVAr. El Statcom entrega 42 MVAr y 170 MVAr en caso base y contingencia, respectivamente.

- Para el escenario 2 hay disminución en generación de seguridad de 147 MW y 160 MVAr. El Statcom entrega 43 MVAr y 155 MVAr en caso base y contingencia, respectivamente.

- Para el escenario 3 hay disminución en generación de seguridad de 55 MW y 187 MVAr. El Statcom entrega 42 MVAr y 200 MVAr en caso base y contingencia, respectivamente.

Con el dato de demanda máxima para la Costa Atlántica en 2018 (2370 MW) y la generación de seguridad para cada escenario (tabla 3), se obtienen los límites de importación aplicando la ecuación (19). Estos límites se reportan en la tabla 4. Note que el uso del Statcom permite importar más potencia en cada uno de los escenarios.

Tabla 4: límites importación para la Costa Atlántica, con y sin Statcom.

\begin{tabular}{|c|c|c|c|c|c|}
\hline \multicolumn{2}{|c|}{ Escenario 1 } & \multicolumn{2}{c|}{ Escenario 2 Escenario 3 } \\
Sin Statcom & Con Statcom & Sin Statcom & Con Statcom & Sin Statcom & Con Statcom \\
\hline 1.956 & 2.037 & 2.030 & 2.174 & 2.060 & 2.129 \\
\hline
\end{tabular}

\subsection{Fase 2: Uso del modelo SDDP para cálculo de factibilidad económica}

Los límites de importación reportados en la tabla 4 se utilizan en el software SDDP para calcular diferencias (beneficios) en costos operativos y de racionamiento, con y sin el Statcom en Caracolí $220 \mathrm{kV}$. Los beneficios se dividen por la inversión (40 USD Millones), para obtener cien relaciones beneficio / costo a ser comparadas con el límite de uno. En la figura 3 se ilustran los resultados del SDDP para los tres escenarios de generación antes descritos. Para los escenarios 1, 2 y 3 el 89\%, 87\% y 70\%, respectivamente, de las series simuladas con el SDDP presentan una relación beneficio / costo mayor o igual a 1,0. Al promediar los resultados de los tres escenarios el $82 \%$ de las series presentó relación beneficio / costo mayor que 1,0 validando la viabilidad económica del proyecto. 


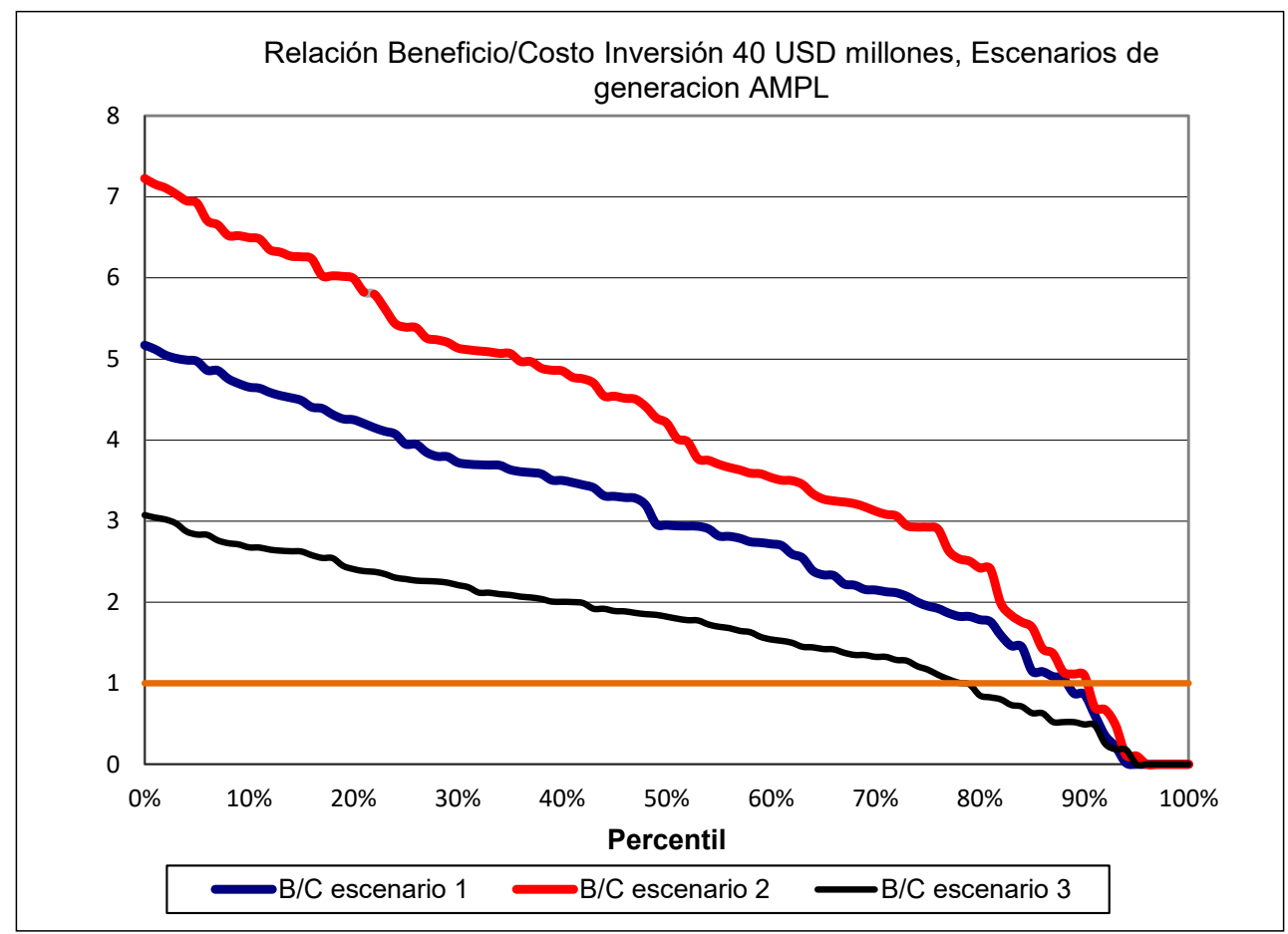

Figura 3: curvas de relación Beneficio/Costo.

Fuente: elaboración propia

\subsection{Validaciones y análisis de flexibilidad ante diferentes escenarios}

Para el escenario de mínima generación de seguridad (escenario 2) se realizaron validaciones, comparando resultados con el software DigSilent Power Factory. Los resultados evidenciaron que las tensiones en las barras estaban dentro de sus límites y no se reportaron problemas de cargabilidad en líneas ni transformadores.

Con el fin de evaluar el comportamiento del Statcom bajo diferentes condiciones operativas, se generaron (de forma aleatoria) otros 9 escenarios de operación. Estos escenarios buscan representar el hecho de que no siempre todas las unidades de generación están disponibles. En la figura 4 se presenta la generación de seguridad para estos escenarios. Los escenarios 1 a 3 son los antes descritos, mientras los escenarios 4 a 12 son nuevos y escogidos de forma aleatoria (los escenarios 7 y 12 resultaron ser infactibles). En la tabla 5 se ilustran los estados operativos de las plantas consideradas en cada escenario. El escenario 5 presenta menor generación de seguridad que los obtenidos por AMPL. Sin embargo, esta solución es descartada ya que requiere diez unidades de generación en funcionamiento (para el escenario 2 solo son necesarias 5 unidades) como se observa en la tabla 5. Todos los resultados mostrados son ante la 
contingencia La Loma-Ocaña $500 \mathrm{kV}$, que se comprobó es la más crítica en el área de la Costa Atlántica.

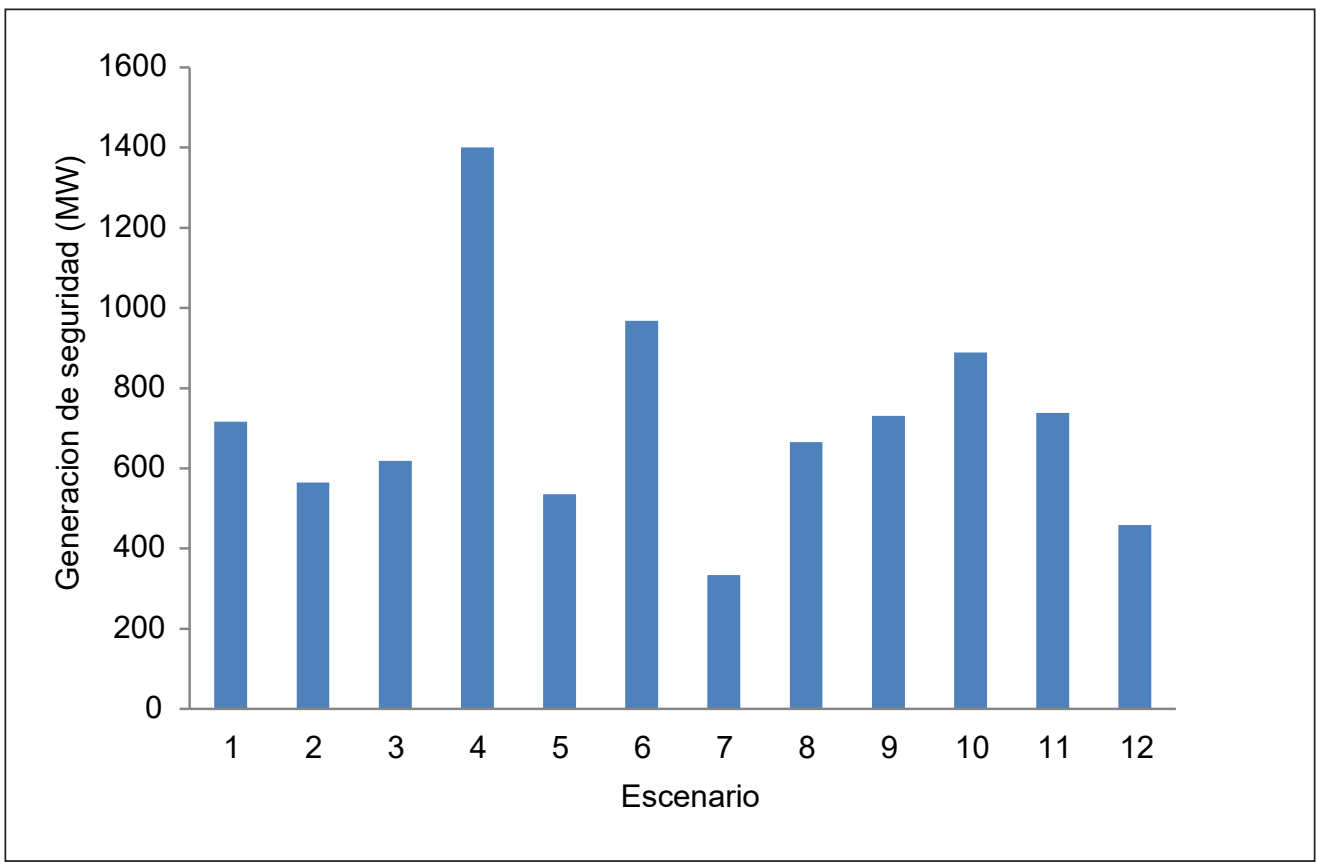

Figura 4: generación de seguridad para diferentes escenarios.

Fuente: elaboración propia

Tabla 5: estado operativo $(\mathrm{On}=1, \mathrm{Off}=0)$ de plantas para cada escenario.

\begin{tabular}{|c|c|c|c|c|c|c|c|c|c|c|c|c|}
\hline \multirow{2}{*}{ Planta } & \multicolumn{12}{|c|}{ Escenario } \\
\hline & 1 & 2 & 3 & 4 & 5 & 6 & 7 & 8 & 9 & 10 & 11 & 12 \\
\hline Barranquilla 3 & 0 & 0 & 0 & 0 & 0 & 0 & 0 & 1 & 0 & 0 & 0 & 0 \\
\hline Barranquilla 4 & 0 & 0 & 0 & 0 & 0 & 0 & 0 & 1 & 0 & 0 & 0 & 0 \\
\hline Guajira 1 & 1 & 0 & 1 & 0 & 0 & 0 & 0 & 1 & 1 & 1 & 1 & 0 \\
\hline Guajira 2 & 0 & 0 & 1 & 0 & 0 & 0 & 0 & 1 & 1 & 1 & 0 & 0 \\
\hline Candelaria 1 & 1 & 1 & 0 & 0 & 0 & 0 & 0 & 1 & 1 & 1 & 1 & 0 \\
\hline Candelaria 2 & 0 & 1 & 0 & 0 & 0 & 0 & 0 & 1 & 1 & 1 & 1 & 0 \\
\hline Cartagena 1 & 0 & 0 & 1 & 0 & 0 & 0 & 0 & 0 & 1 & 0 & 0 & 0 \\
\hline \multirow{2}{*}{ Planta } & \multicolumn{12}{|c|}{ Escenario } \\
\hline & 1 & 2 & 3 & 4 & 5 & 6 & 7 & 8 & 9 & 10 & 11 & 12 \\
\hline Cartagena 2 & 0 & 0 & 0 & 0 & 0 & 0 & 0 & 0 & 1 & 0 & 0 & 0 \\
\hline Flores 1 Gas & 0 & 1 & 0 & 1 & 1 & 1 & 1 & 0 & 0 & 0 & 0 & 0 \\
\hline
\end{tabular}


J. D. Gómez J. - G. E. Vinasco M. - J. M. López-Lezama

\begin{tabular}{|l|c|c|c|c|c|c|c|c|c|c|c|c|}
\hline \multirow{2}{*}{\multicolumn{1}{|c|}{ Planta }} & \multicolumn{10}{|c|}{ Escenario } \\
\cline { 2 - 15 } & 1 & 2 & 3 & 4 & 5 & 6 & 7 & 8 & 9 & 10 & 11 & 12 \\
\hline Tebsa Gas 110 1 & 1 & 1 & 1 & 1 & 1 & 1 & 1 & 0 & 0 & 0 & 0 & 1 \\
\hline Tebsa Gas 110 2 & 0 & 0 & 0 & 1 & 1 & 1 & 0 & 0 & 0 & 0 & 0 & 0 \\
\hline Tebsa Gas 220 1 & 0 & 0 & 0 & 1 & 1 & 1 & 0 & 0 & 0 & 0 & 0 & 0 \\
\hline Tebsa Gas 220 2 & 0 & 0 & 0 & 1 & 1 & 1 & 0 & 0 & 0 & 0 & 0 & 0 \\
\hline Tebsa Gas 220 3 & 0 & 0 & 0 & 1 & 1 & 1 & 0 & 0 & 0 & 0 & 0 & 0 \\
\hline Tebsa Vapor 1 & 1 & 1 & 1 & 1 & 0 & 1 & 1 & 0 & 0 & 0 & 0 & 0 \\
\hline Tebsa Vapor 2 & 0 & 0 & 0 & 1 & 0 & 1 & 0 & 0 & 0 & 0 & 0 & 0 \\
\hline Flores 1 Vapor & 0 & 1 & 0 & 1 & 1 & 1 & 1 & 0 & 0 & 0 & 0 & 1 \\
\hline Flores IV1 & 0 & 0 & 0 & 1 & 1 & 1 & 0 & 0 & 0 & 1 & 1 & 1 \\
\hline Flores IV2 & 0 & 0 & 0 & 1 & 1 & 1 & 0 & 0 & 0 & 1 & 1 & 1 \\
\hline Flores IV3 & 0 & 0 & 0 & 1 & 1 & 1 & 0 & 0 & 0 & 0 & 0 & 0 \\
\hline
\end{tabular}

Fuente: elaboración propia

En la figura 5 se ilustra la cargabilidad de las líneas a $220 \mathrm{kV}$ que en estado operativo normal presentan mayor cargabilidad (según experiencia del operador). Se puede observar que tras la instalación del Statcom los niveles de carga no superan el $56,9 \%$ en todos los escenarios. En la figura 6 se ilustran las tensiones de los nodos más propensos a bajas tensiones ante contingencias (según experiencia del operador). Se observa que las tensiones en estos nodos se mantienen en niveles apropiados. Lo anterior evidencia la efectividad del Statcom.

En la figura 7 se ilustra la inyección de reactivos del Statcom en Caracolí $220 \mathrm{kV}$ ante los escenarios operativos de la figura 4 para la contingencia más crítica.

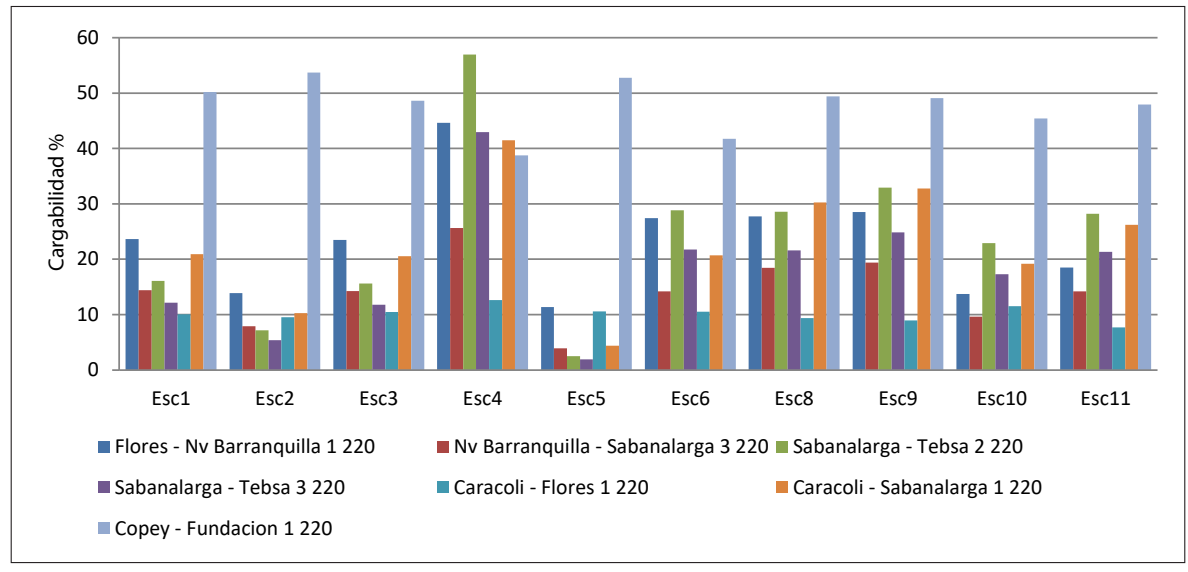

Figura 5: cargabilidad en líneas de 220 kV en contingencia de La Loma-Ocaña 500 kV. 


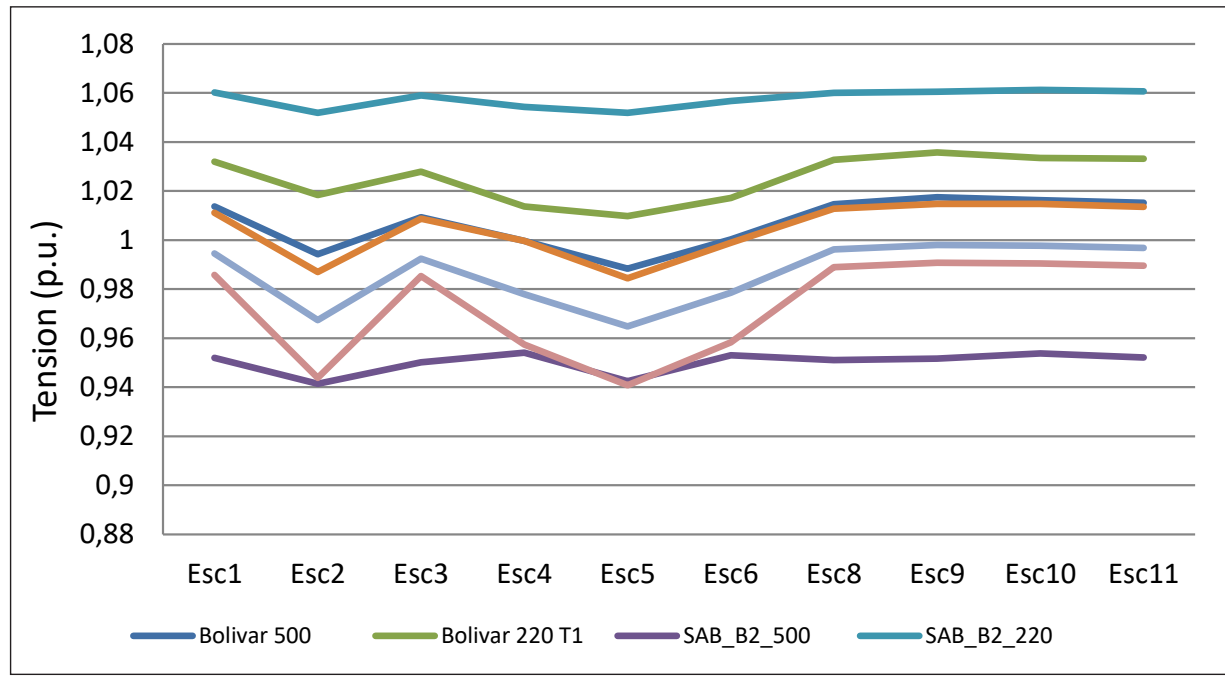

Figura 6: tensiones para diferentes nodos de la red en contingencia La Loma-Ocaña.

Fuente: elaboración propia

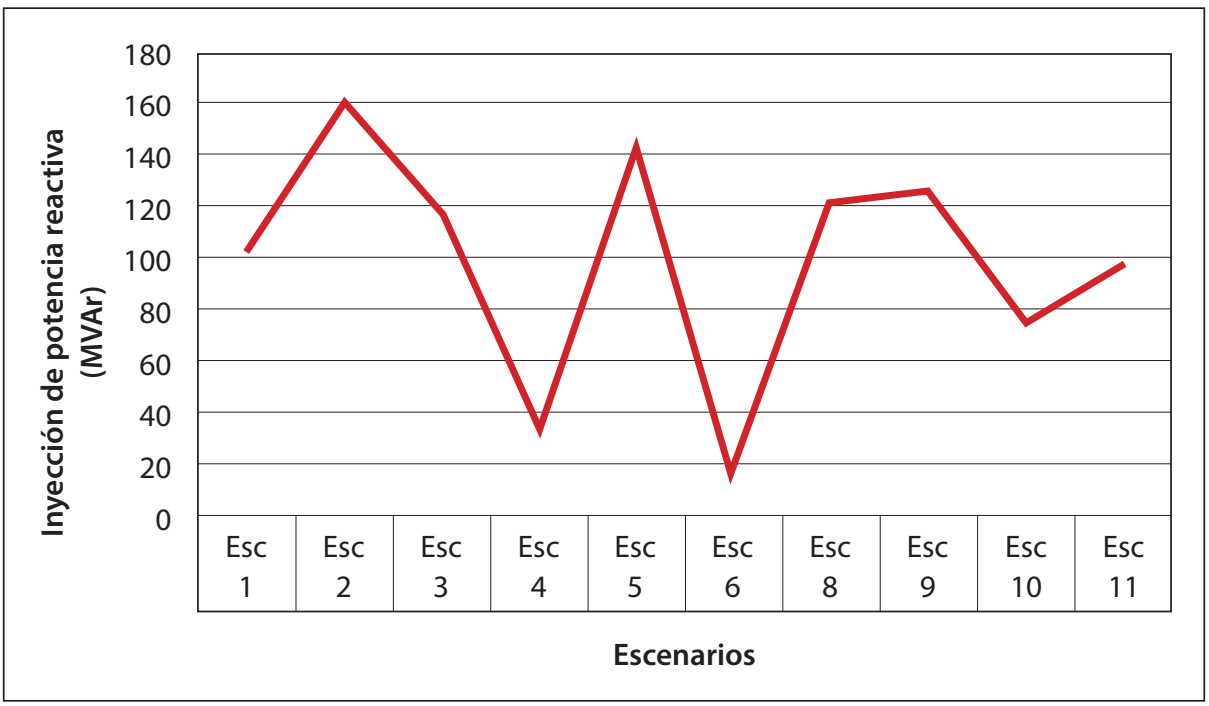

Figura 7: inyección de potencia reactiva del Statcom en contingencia La Loma-Ocaña.

Fuente: elaboración propia

\subsection{Efecto del Statcom en la inestabilidad oscilatoria de tensión}

Posteriores pruebas en DigSilent Power Factory para el escenario 2 mostraron que sin el Statcom en Caracolí $220 \mathrm{kV}$, la contingencia La Loma-Ocaña $500 \mathrm{kV}$ produce inestabilidad oscilatoria de tensión, tal como se ilustra en la figura 8. Este problema 
obtiene solución con el Statcom como se ilustra en figura 9, donde se observa la eliminación de oscilación de tensión.

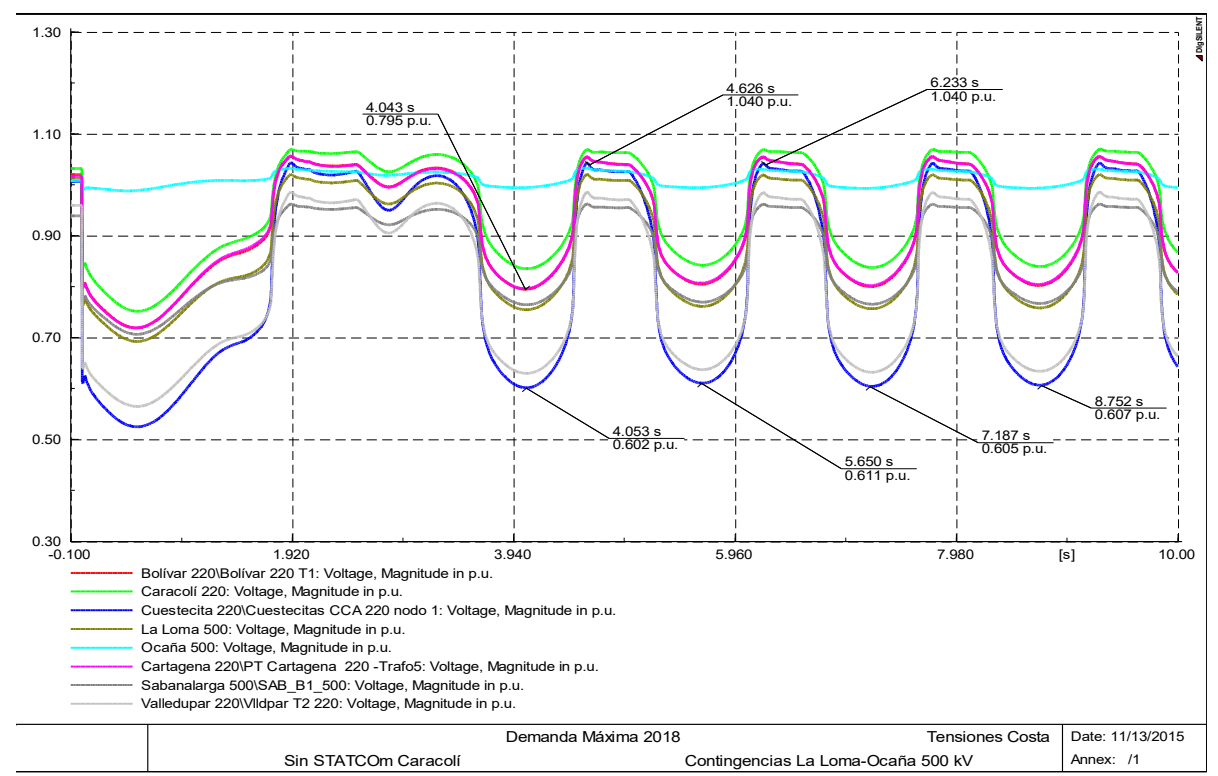

Figura 8: Inestabilidad de tensión en escenario mínimo de generación 2018, contingencia la Loma-Ocaña 500 kV sin Statcom. Fuente: elaboración propia

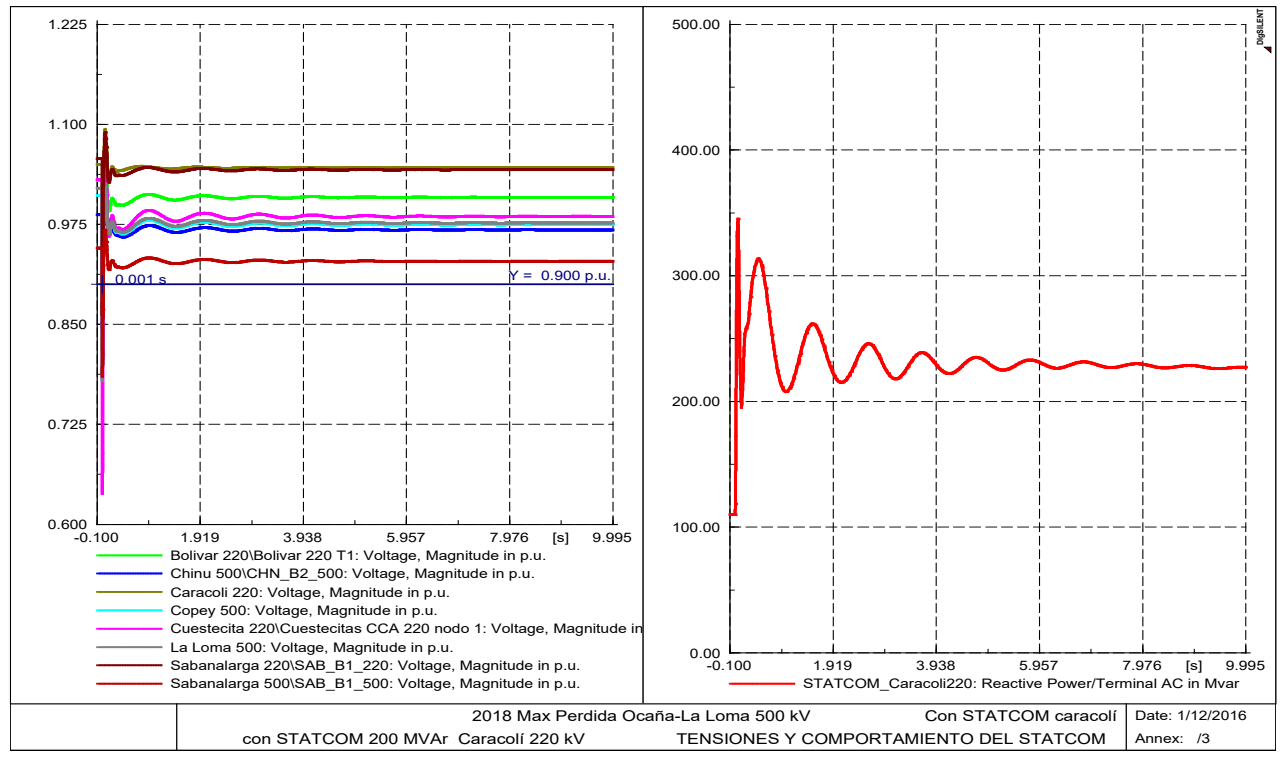

Figura 9: tensiones en nodos y comportamiento del Statcom en Caracolí 220 kV. Fuente: elaboración propia 


\section{CONCLUSIONES}

Se presentó una metodología para evaluar los beneficios técnicos y económicos con la incorporación de Facts en redes de transmisión; esta metodología es aplicable a redes de complejidad y tamaño real.

En una primera fase de la metodología, un modelo eléctrico desarrollado en lenguaje AMPL identifica escenarios de operación seguros, ante la ubicación de dispositivos Facts, considerando contingencias de la red; se optimizan en conjunto: generación de seguridad, taps de los transformadores, operación de la capacidad de los Facts.

En una segunda fase, se presentó cómo incorporar los resultados del modelo eléctrico en AMPL, a un modelo hidrotérmico (SDDP); este último calcula los beneficios económicos, al recibir como dato de entrada el efecto eléctrico de los Facts.

El modelo eléctrico en AMPL fue verificado extensamente mediante la comparación de sus resultados con el programa comercial DigSilent Power Factory.

La metodología anterior fue aplicada al sistema colombiano, un sistema real y complejo para estimar y sustentar mejoras económicas de su operación con dispositivos Facts.

La aplicación anterior de la metodología permitió proponer un proyecto de compensación dinámica (mediante un dispositivo Statcom), que elimina inestabilidad de tensión en la Costa Atlántica que aparece al reducir la generación de seguridad en dicha área para el año 2018.

\section{REFERENCIAS}

[1] S. Elsaiah; N. Cai; M. Benidris y J. Mitra, "Fast economic power dispatch method for power system planning studies," in Generation, Transmission \& Distribution, IET, vol. 9, n. ${ }^{\circ}$, pp. 417-426, 2015.

[2] M. Banzo y A. Ramos, "Stochastic Optimization Model for Electric Power System Planning of Offshore Wind Farms," in Power Systems, IEEE Transactions on, vol. 26, n. ${ }^{\circ}$ 3, pp. 1338$1348,2011$.

[3] E. Ghahremani y I. Kamwa, "Optimal placement of multiple-type Facts devices to maximize power system loadability using a generic graphical user interface," in Power Systems, IEEE Transactions on, vol.28, n. ${ }^{\circ}$, pp. 764-778, 2013.

[4] A. Lashkar Ara, A. Kazemi y S.A.N. Niaki, "Multiobjective Optimal Location of Facts Shunt-Series Controllers for Power System Operation Planning," in Power Delivery, IEEE Transactions on, vol. 27, n. ${ }^{\circ}$ 2, pp. 481-490, 2012. 
[5] E. Ghahremani y I. Kamwa, "Analysing the effects of different types of Facts devices on the steady-state performance of the Hydro-Quebec network," in Generation, Transmission \& Distribution, IET, vol.8, n. ${ }^{\circ}$ 2, pp. 233-249, 2014.

[7] Chao-Ming Huang y Yann-Chang Huang, "Hybrid optimisation method for optimal power flow using flexible AC transmission system devices," in Generation, Transmission \& Distribution, IET, vol.8, n. ${ }^{\circ}$ 12, pp. 2036-2045, 2014.

[6] H. Mehrjerdi; E. Ghahremani; S. Lefebvre; M. Saad y D. Asber, "Authenticated voltage control of partitioned power networks with óptimal allocation of Statcom using heuristic algorithm," in Generation, Transmission \& Distribution, IET, vol. 7, n. ํ 9, pp. 1037-1045, 2013.

[7] K. Balamurugan; G. Suganya y Manojkumar, "Improving power system dynamics by series connected Facts controllers (TCSC)," presented in Power, Energy and Control (ICPEC) International Conference, Sri Rangalatchum Dindigul, 2013.

[8] Xiangjun Li, Dong Hui y Xiaokang Lai, "Battery Energy Storage Station (BESS)-Based Smoothing Control of Photovoltaic (PV) and Wind Power Generation Fluctuations," in Sustainable Energy, IEEE Transactions on, vol. 4, n. ${ }^{\circ}$ 2, pp. 464-473, 2013.

[9] S. Chansareewittaya y P. Jirapong, "Optimal allocation of multi-type Facts controllers for total transfer capability enhancement using hybrid particle swarm optimization," presented in Electrical Engineering/Electronics, Computer, Telecommunications and Information Technology (ECTI-CON International Conference, Nakhon Ratchasima, 2014.

[10] Y. Sonmez; S. Duman; U. Guvenc y N. Yorukeren, "Optimal power flow incorporating Facts devices using Gravitational Search Algorithm," presented in Innovations in Intelligent Systems and Applications (INISTA) International Symposium, Trabzon, 2012.

[11] S. Gerbex; R. Cherkaoui; y Aj., Germond; "Optimal location of multi-type Facts devices in a power system by means of Genetic Algorithms." IEEE Transactions on Power Systems, vol. 16, n. 3 , pp. 537-544, 2001.

[12] N. Rawat; A. Bhatt y P. Aswal, "A review on optimal location of Facts devices in AC transmission system," presented in Power, Energy and Control (ICPEC) International Conference, Sri Rangalatchum Dindigul, 2013.

[13] F. Robert, D. M. G. y W. Brian, "manual de usuario para AMPL” [en línea], disponible: http:// ampl.com/BOOK/CHAPTERS/24-refman.pdf, 2003.

[14] R. Otalvaro, G. Vinasco.; "Localización Óptima de Statcom considerando Contingencias en la red", Revista AIE U. de A., n. ${ }^{\circ}$ 14, pp. 47-53, 2015.

[15] PSR, “SDDP Manual del Usuario, versión 12.0”, PSR [en línea], Disponible: http://www. psr-inc.com/psr/download/manual/SddpUsrEsp.pdf, 2013.

[16] DigSilent Power Factory, "Manual de usuario DigSilent" [en línea], disponible: http://www. digsilent.de/tl_files/digsilent/files/powerfactory/PowerFactory15/WhatsNew_PF150.pdf, 2012.

[17] O. Megel, J.L. Mathieu y G. Andersson, "Stochastic Dual Dynamic Programming to schedule energy storage units providing multiple services," presented in PowerTech, International conference, Eindhoven, 2015. 